SISTEMA HIPERMEDIA ADAPTATIVO PARA EL APRENDIZAJE DE BÚSQUEDAS AVANZADAS DE INFORMACIÓN $\quad$ EN Eduardo Martínez y Any Montero ( PP. 81-107)

DE BARQUSIMETO
LUIS BELTRAN PRIETO FIGUEROA

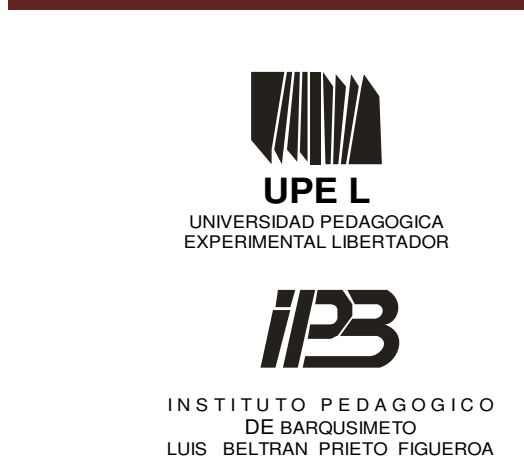

(1)

.

.

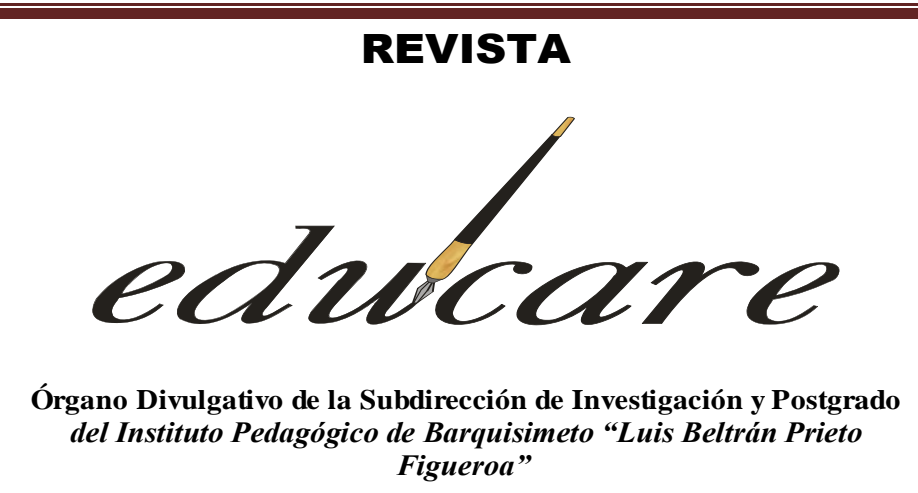

Figueroa”

\author{
BARQUISIMETO - EDO. LARA - VENEZUELA \\ NUEVA ETAPA \\ FORMATO ELECTRÒNICO \\ DEPOSITOLEGAL: ppi201002LA3674
}

ISSN: 2244-7296
Volumen 20 № 1

Enero - Abril 2016

\title{
SISTEMA HIPERMEDIA ADAPTATIVO PARA EL APRENDIZAJE DE BÚSQUEDAS AVANZADAS DE INFORMACIÓN EN INTERNET
}

\author{
Eduardo Martínez* \\ Any Montero *
}

\section{* UNIVERSIDAD PEDAGÓGICA EXPERIMENTAL LIBERTADOR - INSTITUTO PEDAGÓGICO DE BARQUISIMETO (UPEL-IPB)}




AVANZADAS DE INFORMACIÓN $\quad$ EN $\quad$ INTERNET

Eduardo Martínez y Any Montero ( PP. 81-107)

\section{SISTEMA HIPERMEDIA ADAPTATIVO PARA EL APRENDIZAJE DE BÚSQUEDAS AVANZADAS DE INFORMACIÓN EN INTERNET}

TRABAJO DE INVESTIGACIÒN

Recibido: 25/11/2015

\author{
Eduardo Martínez * \\ Any Montero** \\ UPEL IPB \\ Aceptado 20/02/2016
}

\section{RESUMEN}

Este estudio tuvo como objetivo diseñar un software educativo para el aprendizaje de búsquedas avanzadas de información en Internet, dirigido a los estudiantes de la UCLA. La metodología se fundamentó en la modalidad de proyecto especial desarrollado en tres (3) fases: Fase I: Estudio diagnóstico, sustentado en una investigación de campo de tipo descriptivo. Para la recolección de datos se utilizó la técnica de la encuesta y un cuestionario de opinión con múltiples alternativas de respuesta, aplicado a una muestra aleatoria de 30 estudiantes pertenecientes a la población objeto de estudio. La validez de contenido se realizó a través de la técnica de juicio de expertos, y la confiabilidad con el método de consistencia interna, con un resultado $\alpha=0,88$. La información recolectada se analizó e interpretó por medio de estadísticos descriptivos a través de frecuencias, porcentajes y gráficas. Los resultados del estudio comprobaron la pertinencia del diseño propuesto. En la Fase II, fue diseñado el Software sobre la base del modelo MOOHM propuesto por Benigni, para su implementación se utilizó Microsoft Visual Studio. La Fase III, comprendió la validación del Software Educativo a través de expertos y usuarios, utilizando (4) instrumentos adaptados del modelo propuesto por Marquès. El diseño e implementación del Software redundará en el desarrollo de competencias en los estudiantes para la búsqueda pertinente de información.

Descriptores: Software educativo, Sistema Hipermedia Adaptativo, Búsquedas de Información en Internet

\begin{abstract}
This study aimed to design an educational software for advanced information search learning on the Internet, aimed at UCLA students. A special project methodology was developed in three (3) phases: Phase I: Diagnostic study, based on a descriptive field research. For data collection, the survey technique was used and an opinion questionnaire with multiple response alternatives, applied to a random sample of 30 students belonging to the study population. The validity of content was made through the technique of expert judgment, and reliability with the method of internal consistency, with a result $\alpha=0.88$. The information collected was analyzed and interpreted through descriptive statistics through frequencies, percentages and graphs. The results of the study verified the relevance of the proposed design. In Phase II, the software was designed based on the MOOHM model proposed by Benigni, the Microsoft Visual Studio was used for its implementation. Phase III included the validation of the Educational Software through experts and users, using (4) adapted instruments of the model proposed by Marqués. The design and implementation of the Software will result in the development of competences in the students for the pertinent search of information.
\end{abstract}

Keywords: Educational Software, Adaptive Hypermedia System, Internet Search Information

*Magister en Investigación Educacional. Cursante del Doctorado en Educación PIDE (UCLA-UPELUNEXPO). Correo Electrónico: eduardoemg1@gmail.com

** Doctora en Educación PIDE (UCLA-UPEL-UNEXPO). Docente adscrita al Departamento de Formación Docente de la UPEL IPB. Coordinadora General de Postgrado UPEL IPB. Correo Electrónico: asmonterosopilca@hotmail.com 
SISTEMA HIPERMEDIA ADAPTATIVO PARA EL APRENDIZAJE DE BÚSQUEDAS AVANZADAS DE INFORMACIÓN $\quad$ EN Eduardo Martínez y Any Montero ( PP. 81-107)

\section{INTRODUCCIÓN}

En la presente Era, el uso masivo de los medios electrónicos, para difundir e intercambiar la información que circula por todo el planeta, hace que la misma se convierta en el insumo fundamental que impulsa el desarrollo global de la denominada Sociedad de la Información (Godoy, 2010). Esta a su vez, concibe un entorno dinámico, fundamentado en las Tecnologías de Información y Comunicación (TIC), al promover cambios vertiginosos en los modos de vivir, aprender y trabajar de la sociedad.

En este sentido, las TIC han penetrado en todos los campos de aplicación y planos del conocimiento humano. Particularmente en el terreno de la educación han sido intensamente utilizadas, y de diferentes maneras, con la finalidad de apoyar los procesos realizados en el área. Por lo que, su uso posee un papel trascendental, a través de la incorporación de computadoras en los diferentes ámbitos, incluyendo formación, en todos los niveles.

Asimismo, la utilización de las computadoras en el ámbito educativo han brindado nuevos horizontes en el proceso de formación, permitiendo el diseño y desarrollo de sistemas conocidos como Software Educativo, es decir, herramientas informáticas que potencian una nueva concepción de los procesos de aprendizaje, en la que el docente y el estudiante se favorecen de gran manera.

De acuerdo con Benigni y Marcano (2014a), el software educativo "es una aplicación informática, que construido sobre una base pedagógica bien definida, puede constituirse en un apoyo docente durante el proceso de enseñanza aprendizaje de lo contrario, podría llegar a considerarse un elemento distractor en el proceso"(p.298). Por lo que, para que potencien el proceso de aprendizaje, se requiere de sistemas de información tecnológicos que permitan una personalización y adaptación de la información a las necesidades específicas de aprendizaje de cada estudiante.

Actualmente, los Sistemas Hipermedia Adaptativo Educativo (SHAE) representan una de las técnicas con mayor aceptación para la de desarrollos informáticos orientados a la personalización del aprendizaje, ya que proporcionan muchas de las características necesarias en el campo educativo computacional, como son la interactividad, el uso de grandes bases de información, la información multimedia, y la representación del conocimiento de forma similar a la forma de procesamiento de la información del estudiante (Vera, Cerón, Navarro y Ortiz, 2014). 
SISTEMA HIPERMEDIA ADAPTATIVO PARA EL APRENDIZAJE DE BÚSQUEDAS AVANZADAS DE INFORMACIÓN EN

Eduardo Martínez y Any Montero ( PP. 81-107)

En este orden de ideas, el desarrollo cada vez más avasallante de la Internet, aunado con los estímulos que reciben los estudiantes a través de todos sus servicios, que les invitan a acceder a información proveniente de cualquier parte del globo terráqueo. No cabe duda, de acuerdo con Cornella (citado por, Godoy, 2010) que las TIC es el principal causante de un fenómeno denominado infoxicación, es decir, la sobrecarga de información. Por lo tanto, en el presente siglo, las universidades venezolanas, no escapan de este hecho, así que, se requiere de un uso, con destreza y creatividad de las herramientas informáticas para superar el suceso mencionado.

En tal sentido, el SHAE se presenta como una de las mejores opciones, dentro de la tecnología informática, para el diseño de Software Educativos que garanticen los requerimientos de información de una población de usuarios grande y heterogénea. Por lo que, en el ámbito del presente estudio, este tipo de sistemas adaptará el aprendizaje del proceso de Búsquedas Avanzadas de Información en Internet de los estudiantes del primer semestre de Ingeniería Agronómica de la Universidad Centroccidental Lisandro Alvarado (UCLA). Por tal motivo, el propósito de esta investigación es el desarrollo de un Software Educativo para el Aprendizaje de Búsquedas Avanzadas de Información en Internet.

\section{PLANTEAMIENTO DEL OBJETO DE ESTUDIO}

La sociedad de la información ultimamante favorecida por los continuos avances científicos y tecnológicos, impulsa de manera constante una tendencia hacia la globalización económica y cultural de las naciones. Esta cambiante humanidad cuenta con una difusión masiva de información a través de diversos medios de comunicación. De igual manera,organiza, procesa y transmite la misma en formato digital a través de las Tecnologías de Información y Comunicación (TIC) (Marquès, 2007).

En este sentido, las TIC han originado una globalización tecnológica que ha proporcionado un acceso fácil y económico a una gran diversidad de información, desde cualquier lugar del mundo,impulsando la conectividad de las sociedades a nivel internacional. Es decir, se han convertido en un medio que refleja las presentes tendencias comunicacionales de la estructura social de los países.

Asimismo, la transformacióndelos distintos aspectos del proceso de globalización se diferencia de épocas anteriores,en relacióna su alcance, rapidez y complejidad.Por lo tanto, esta 
SISTEMA HIPERMEDIA ADAPTATIVO PARA EL APRENDIZAJE DE BÚSQUEDAS AVANZADAS DE INFORMACIÓN

Eduardo Martínez y Any Montero ( PP. 81-107)

renovación organizacional ha sentado las bases de un nuevo paradigma tecnológico que ha innovado las estructuras y relaciones sociales,esta ha sido denominada segúnel sociólogo Castell (2009) “La Era de la Información”.

En este aspecto, la Era de la Información constituye un período en el cual los contenidos digitales se procesan de forma más rapida que en cualquier otra época, esto se debe a la llegada de tecnologías que brindan mayor flexibilidad y movilidad,permitiendo la realización de cualquier actividad desde distintos lugares. Por lo tanto, es importante destacar el papel de Internet como la herramienta que constituye el cimiento de esta época tecnológica.

Asimismo, este desarrollo dinámico y sostenido en relación a los contenidos publicados en el ciberespacio ha provocado la aparición de un factor mucho más importante que la conectividad como elemento de división social. Este se refiere a la capacidad educativa y cultural de utilizar la información divulgada enla Internet, en palabras del citado autor,

Una vez que toda la información está en la red, una vez que el conocimiento está en la red, el conocimiento codificado, pero no el conocimiento que se necesita para lo que se quiere hacer, de lo que se trata es de saber dónde está la información, cómo buscarla, cómo procesarla, cómo transformarla en conocimiento específico para lo que se quiere hacer (Castell, ob.cit., p.5).

Resulta claro para el referido autor, que la capacidad educativa y cultural del uso de Internet, depende inicialmente de saber cómo buscar la información, en otras palabras, es importante saber cómo encontrarla para luego procesarla y transformar la en un conocimiento aplicado a un contexto específico. Esta afirmación permite destacar que el proceso de investigación en Internet resulta de suma importancia para potenciar el proceso social educativo de las naciones.

Del mismo modo Calderín y Rojano (2006), destacan que hasta hace muy poco tiempo la difusión de la ciencia se limitaba al formato impreso, o la transmisión oral, pero en esta sociedad se presenta Internet como un nuevo esquema de comunicación y acceso al conocimiento humano. Es decir, impera un consumo creciente y acelerado de la información, lo que demanda el desarrollo de habilidades y destrezas en materia de búsqueda y recuperación de recursos Web, especialmente en el proceso educativo.

Por consiguiente, en el ámbito académico, la demanda de fuentes de información confiables en Internet se ha transformado en un proceso cada vez más complejo que requiere de un conocimiento exhaustivo. Esto se debe a la saturación de contenidos en los motores de 
SISTEMA HIPERMEDIA ADAPTATIVO PARA EL APRENDIZAJE DE BÚSQUEDAS AVANZADAS DE INFORMACIÓN

Eduardo Martínez y Any Montero ( PP. 81-107)

búsqueda, así como también por las páginas Web que contienen artículos relevantes que no son accesibles de forma inmediata. Por lo tanto, esto dificulta la localización de documentación significativa en el ciberespacio que fundamente las argumentaciones académicas del entorno educativo.

En relación con lo planteado, se puede citar que en la universidad de Alcalá de España, desde el 2012, el Departamento de Ciencias de la Computación ofrece una Asignatura llamada "Búsqueda y Gestión de la Información y Recursos Multimedia", la cual asume como propósito brindarles a los estudiantes la posibilidad de convertirse en especialistas en el uso de herramientas de búsqueda y recuperación de la información en la Internet, pues lo considera imprescindible para un desempeño satisfactorio de su labor académica y profesional.

A nivel latinoamericano, específicamente en Argentina, el Programa "Conectar Igualdad", creado por decreto del gobierno nacional de ese país en 2013, surge como una política destinada a favorecer la inclusión social y educativa a partir de acciones que aseguren el acceso y promuevan el uso de las TIC en la educación. Dentro de ese programa destaca el de “Investigación, Gestión y Búsqueda de Información en Internet”. Este tiene como propósito que el estudiante desarrolle competencias que le permitan acceder al gran cúmulo de contenidos de la Internet, mediante técnicas que le permitan encontrar lo que está buscando. Además de determinar si la información que alcanza es pertinente y confiable.

Dentro de este mismo orden de ideas, en la Universidad de Chile desde el 2009, se imparte una asignatura de formación general llamada "Información Académica en Internet: Búsqueda Eficaz y Uso Ético". Este curso está dirigido a los estudiantes de las Facultades: Ingeniería, Medicina, Química, Ciencias, Economía, Periodismo, y tiene como propósito posicionar a los estudiantes en un proceso de adquisición y generación de conocimiento para promover activamente en ellos las habilidades tecnológicas como la capacidad de localizar, evaluar y utilizar eficazmente la información requerida.

Es conveniente señalar, que en Venezuela la Universidad Católica Andrés Bello a través de su Centro de Investigación de la Comunicación en el Área de las TIC, presenta en 2010el programa Sistemas de Información Documental en Comunicación Social con el propósito de promover la competencia informacional "Estrategias y herramientas de búsqueda y recuperación en la Web”. En otras palabras, atiende la alfabetización en información a través de la creación de una habilidad genérica con miras a proporcionar un conjunto de aptitudes para 
SISTEMA HIPERMEDIA ADAPTATIVO PARA EL APRENDIZAJE DE BÚSQUEDAS AVANZADAS DE INFORMACIÓN $\quad$ EN INTERNET Eduardo Martínez y Any Montero ( PP. 81-107)

localizar, manejar y utilizar los recursos en la Internet de forma eficaz para una gran variedad de finalidades.

Desde la perspectiva que se ha venido desarrollando, se hace necesario que tanto el docente como el estudiante se involucren en un proceso de aprendizaje acorde con las tendencias actuales de las TIC en la educación, de modo que se promueva el fortalecimiento de habilidades en el manejo de herramientas para la recuperación de información en Internet, promoviendo de este modo el acceso a la gran diversidad de recursos existentes en la red de manera exitosa, en cuanto a la efectividad de los resultados obtenidos.

En relación a las TIC, se presenta un campo extenso y de mucho interés para el proceso de aprendizaje, entre estos, se encuentra el uso del Software Educativo Inteligente:

Las tendencias a nivel mundial en el uso de las Tecnologías de la Información y Comunicación aplicadas en contextos educativos, plantean el desarrollo desoftware con Inteligencia Artificial. En este campo de creciente interés se trata, fundamentalmente, de aplicar las técnicas de la Inteligencia Artificial al desarrollo de sistemas de enseñanza por computadora con el propósito de construir sistemas educativos inteligentes (Barragán, 2008, p.3).

Los sistemas educativos inteligentes poseen ciertas características importantes para los entornos de aprendizaje, como es la capacidad de adaptación a través de los estilos de aprendizaje del estudiante. Por otra parte, se diferencian del software educativo tradicional que aborda de manera rutinaria la problemática educativa. Con el auge del uso del Software Educativo vía Internet surgen los Sistemas Hipermedia Adaptativos Educativos (SHAE), que nacen de los Sistemas Hipermedias y de los Sistemas Educativos Inteligentes, con el propósito fundamental de construir nuevos escenarios académicos acordes con las nuevas tecnologías.

El propósito de un SHAE de acuerdo con Grimón, Guevara y Monguet (2010) es construir un espacio de aprendizaje que se pueda ajustar a las particularidades de cada estudiante, logrando así configurar entornos educativos modernos. Esto permite desarrollar un sistema con preferencias y conocimientos de un estudiante específico, es decir, que la interacción dependerá de sus características y necesidades de aprendizaje.

Cabe destacar, que la importancia de los estilos de aprendizaje en el mejoramiento de la calidad de la educación ha sido una tendencia en la investigación desde los últimos años. En un estudio realizado por Soler (2007) revelo que "el aprendizaje depende de varios factores personales y que prácticamente todo individuo posee un estilo propio, además, éste no siempre 
SISTEMA HIPERMEDIA ADAPTATIVO PARA EL APRENDIZAJE DE BÚSQUEDAS AVANZADAS DE INFORMACIÓN AN

Eduardo Martínez y Any Montero ( PP. 81-107)

permanece invariable sino que puede cambiar con el tiempo y depender del contexto de las tareas educativas" (p.10). Es allí, donde los Sistemas Hipermedia Adaptativos cobran gran importancia para el entorno académico, pues, permiten el desarrollo y aplicación de las teorías de aprendizaje pertenecientes a las corrientes pedagógicas más modernas.

El desarrollo de los SHAE en el país específicamente dentro del sector universitario es reducido, esto se puede constatar empíricamente en el Decanato de Agronomía de la Universidad Centroccidental Lisandro Alvarado (UCLA).Por lo tanto ante la situación planteada, el software basado en estilos de aprendizaje se presenta como una opción potencial para abordar la carencia de este tipo de herramienta tecnológica en el mencionado entorno universitario.

Resulta oportuno resaltar, que los estudiantes de Ingeniería Agronómica de la UCLA han manifestado por medio de conversaciones informales que utilizan cada vez con mayor frecuencia el Internet para investigar sobre cualquier tema académico de interés para su carrera; sin embargo no disponen de ningún curso o software como medio de aprendizaje sobre las búsquedas de información en Internet.

En relación con esto último, se observa empíricamente en el laboratorio de computación del Programa de Ingeniería Agronómica de la UCLA, que los estudiantes del 1er semestre utilizan de manera básica el buscador de información Google, no toman en consideración que los resultados obtenidos son ordenados por el ranking de páginas más visitadas, y no por la conformidad con el tema investigado. En consecuencia, docentes del mismo Decanato de la Universidad han manifestado por medio de entrevistas informales que los resultados obtenidos por sus estudiantes discrepan de la necesidad de información de este entorno universitario, demandante de fuentes académicas, científicas, y acreditadas del conocimiento.

De acuerdo con lo anterior, docentes adscritos al mismo Decanato han manifestado que se presenta una discrepancia entre la información consultada por los estudiantes y la información requerida para satisfacer las necesidades académicas de investigación universitaria. Esto, según ellos, se debe a que la información utilizada como base bibliográfica para la elaboración de asignaciones académicas provenga de fuentes de Internet no fiables, es decir, sitios web sin ninguna finalidad académica, científica o especializada.

En este sentido, los mismos docentes han expresado disconformidades en relación a los resultados del proceso de búsqueda realizado por los estudiantes. Es decir, alegan que la 
SISTEMA HIPERMEDIA ADAPTATIVO PARA EL APRENDIZAJE DE BÚSQUEDAS AVANZADAS DE INFORMACIÓN Eduardo Martínez y Any Montero ( PP. 81-107)

información se encuentra descontextualizada, pues proviene de otras ubicaciones geográficas distintas al tema de interés; asimismo, ocurre cuando la información se obtiene de forma desactualizada, en otras palabras, ha perdido vigencia académica; y por último, también se producen resultados incoherentes cuando la información recabada es insuficiente, debido a que se consiguen pocos resultados pertinentes a las exigencias académicas.

En tal sentido, el diseño de un Sistema Hipermedia Adaptativo para el Aprendizaje de Búsquedas Avanzadas de Información en Internet, dirigido a los estudiantes del 1er semestre de Ingeniería Agronómica de la UCLA. Pretende dar una respuesta a la problemática planteada.

\section{OBJETIVOS DEL ESTUDIO}

Diagnosticar la necesidad del diseño de un Sistema Hipermedia Adaptativo para el Aprendizaje de Búsquedas Avanzadas de Información en Internet.

Diseñar el Sistema Hipermedia Adaptativo para el Aprendizaje de Búsquedas Avanzadas de Información en Internet que exponga las diversas formas de localización de recursos en la Web.

Validar el Sistema Hipermedia Adaptativo para el Aprendizaje de Búsquedas Avanzadas de Información en Internet a través de potenciales usuarios y de expertos.

\section{ARGUMENTACIÓN TEÓRICA}

A continuación se desglosa las consideraciones teóricas más relevantes que le dan apoyo y fortaleza al presente estudio

\section{Tecnologías de la Información y Comunicación}

Las Tecnologías de la Información y Comunicación (TIC) se definen por una asociación de distintas tecnologías, producto del avance del conocimiento científico, que permiten procesar, almacenar, transmitir, gestionar, digitalizar, codificar, comunicar datos e información. Su clasificación, abarca desde las transmisoras, interactivas y colaborativas. Estas se enfocan en: las telecomunicaciones, computadora y vídeo interactivo, las cuales pueden ser utilizadas ampliamente en las distintas modalidades de estudio (Ortiz ,2002).

Sin embargo, las TIC abarcan más que una definición, es importante estudiar sus características para aprender a diferenciarlas de las tecnologías tradicionales, con el propósito 
SISTEMA HIPERMEDIA ADAPTATIVO PARA EL APRENDIZAJE DE BÚSQUEDAS AVANZADAS DE INFORMACIÓN

Eduardo Martínez y Any Montero ( PP. 81-107)

de emplearlas a cabalidad especialmente en el campo educativo. Para ello, Cabero (Citado por Cañellas, 2006) realiza un análisis donde muestra sus elementos influyentes, estos se resumen en: (a) Inmaterialidad, su materia prima es la información en formato digital, en múltiples códigos y formas: visuales, auditivas, audiovisuales, textuales, de datos. (b) Interconexión, aunque suelen presentarse de forma independiente, ofrecen grandes posibilidades para que puedan combinarse y ampliar de esta forma sus potencialidades y extensiones. (c) Interactividad, es una de las características que le permiten adquirir un sentido pleno en el terreno de la formación, y que permite una interacción sujeto y computadora; (d) La instantaneidad, facilita la rapidez al acceso e intercambio de información, rompiendo las barreras espacio temporales que han influido durante bastante tiempo la organización de actividades formativas. Es importante mencionar que las TIC promueven la creación de nuevos lenguajes comunicativos que permiten nuevas realidades expresivas como es el caso de los multimedia e hipertextos, y al mismo tiempo conllevan a la necesidad de adquirir nuevos dominios alfabéticos y la posibilidad de la interconexión de las mismas. Así como la tendencia progresiva a la automatización y el desarrollo de la Autonomía e iniciativa constante por parte de los usuarios. Es decir, se produce la autonomía en la búsqueda de información, aunque en principio necesite aprender a utilizarla y seleccionarla.

\section{Software educativo}

García (2004) define el software educativo como, programa que transforma a la computadora en una máquina con fines académicos, lo que significa, capaz de propiciar el desarrollo de competencias, procedimientos y aprendizajes en un contexto determinado y que tiene en cuenta las características del grupo de personas al que va dirigido.

Es decir, es un medio fundamental para la interacción en el que convergen las características del mismo a través de sus dimensiones educativas e instructivas. Así mismo, diferentes han sido los criterios de los especialistas al referirse a su definición, entre los más renombrados, pero no los únicos, están: Pere Marquès, Begoña Gros, Silva Sánchez, Gerson Berrios y Galvis (Citados por García, ob.cit.).

Todos los autores anteriormente citados concuerdan en que: (a) Son materiales elaborados con una finalidad didáctica, como se desprende de la definición; (b) Utilizan el computador como soporte en el que los estudiantes realizan las actividades que se proponen,; 
SISTEMA HIPERMEDIA ADAPTATIVO PARA EL APRENDIZAJE DE BÚSQUEDAS AVANZADAS DE INFORMACIÓN A

Eduardo Martínez y Any Montero ( PP. 81-107)

(c) Son interactivos, contestan inmediatamente las acciones de los estudiantes y permiten un diálogo y un intercambio de información entre los mismos y el computador; (d) Individualizan el trabajo de los estudiantes, ya que se adaptan al ritmo de trabajo de cada uno y pueden adaptarse a sus actividades según las actuaciones de los mismos; (e) Son amigables, y fáciles de usar, con controles de uso deducible sin mayores problemas y sin requerir experiencia previa.

En definitiva, el software educativo al igual que otros medios didácticos, en última instancia tiene como función convertirse en vehículos que faciliten el proceso de formación educativo.

\section{Clasificación de Software Educativo}

Galvis (Citado por Salcedo, 2000), plantea la siguiente clasificación de Software Educativo: Los sistemas tutoriales, sistemas de ejercitación y práctica, simuladores, juegos educativos, sistemas expertos y sistema tutorial inteligente. Este último presenta un comportamiento inteligentemente adaptativo, es decir, el proceso educativo se establece en función de aquello que se desea aprender en conjunto con las características y desempeño del aprendiz.

Para el presente estudio, se empleó las tecnologías adaptativas e inteligentes como propuesta para el diseño del Software Educativo para el Aprendizaje de Búsquedas Avanzadas de Información en Internet. Apoyándose en lo propuesto por Brusilovsky, en relación a la tendencia evolutiva de los Sistemas Tutoriales Inteligentes que los ha llevado a integrarse en la Web, siendo los Sistemas Hipermedia Adaptativos un claro resultado de este acercamiento (Citado por García, 2004).

\section{Sistema Hipermedia Adaptivo Educativo}

De acuerdo con Brusilovsky (citado por Benigniy Marcano, 2014b) los Sistemas Hipermedia Adaptivos (SHA) se derivan a partir de la integración de un sistema hipermedia (SH) con un sistema de tutor inteligente (STI), lo que permite incorporar las ventajas de ambos tipos de software, como por ejemplo, un SH se caracteriza por la libertad de acceso a los contenidos, mientras un STI controla al usuario y adapta el sistema. Por lo tanto, el STI aprovecha la flexibilidad y utilización de distintos medios audiovisuales que motivan al usuario 
SISTEMA HIPERMEDIA ADAPTATIVO PARA EL APRENDIZAJE DE BÚSQUEDAS AVANZADAS DE INFORMACIÓN

Eduardo Martínez y Any Montero ( PP. 81-107)

y proporcionan distintas formas de presentar la información que provee el $\mathrm{SH}$, y este último a la vez se favorece de la adaptación al usuario que realiza el STI.

La hipermedia adaptativa surge como área de investigación dentro del campo de la hipermedia con el objetivo de incrementar la funcionalidad de la misma, de modo que, tanto la forma de presentar la información como la propia que es presentada, se adapten a las necesidades de cada usuario concreto. Para conseguir este objetivo, el autor antes mencionado señala que los sistemas hipermedia adaptativos construyen un modelo con las características personales de cada usuario y lo utilizan durante la interacción del mismo con el sistema.

El entorno educativo ha sido una de las aplicaciones más populares en el área de los SHA. No solo se han realizado muchas investigaciones en este campo, sino que además las técnicas y métodos fueron desarrollados en un principio para el mejoramiento del proceso educativo. A través de ellos se puede guiar al estudiante de manera personalizada en su proceso de estudio y de experimentación de forma que se puede lograr un aprendizaje más eficiente (Grimón, 2008).

Asimismo, existen diversas definiciones sobre los SHA, por lo que a continuación son presentadas algunas de las más destacadas por su aceptación en el área educativa. Para Gaudioso (citado por Benigni y Marcano, ob.cit.)son aquellos capaces de ajustar su presentación y navegación a las diferencias de los usuarios, reduciendo así los problemas de desorientación y falta de comprensión, propios de los sistemas hipermedia no adaptativos. Por lo que, estos entornos que se basan en hipertexto e hipermedia reflejan algunas características del participante en el modelo de usuario y lo aplican para adaptar varios aspectos visibles del sistema al mismo.

En este mismo orden de ideas, los SHA tiene como función personalizar el proceso de aprendizaje con la intención de facilitar la adquisición del conocimiento, presentando contenidos educativos y recorridos apropiados a las metas educativas, formación previa, características individuales o nivel de conocimiento de cada estudiante (Berlanga, 2006).Asimismo, (Benigni y Marcano, ob.cit.) la naturaleza de los sistemas hipermedia educativos adaptativos, estos pueden generar mayores beneficios como herramientas de apoyo en el proceso aprendizaje, debido al valor agregado que éstos tienen al mantener un ritmo de interacción particular según el usuario. 
SISTEMA HIPERMEDIA ADAPTATIVO PARA EL APRENDIZAJE DE BÚSQUEDAS AVANZADAS DE INFORMACIÓN $\quad$ EN INTERNET Eduardo Martínez y Any Montero ( PP. 81-107)

Para el presente estudio un "Sistema Hipermedia Adaptativo" (SHA) asumirá un propósito educativo, de acuerdo con Arteaga (2003) este satisface necesidades de aprendizaje, la presentación de la información está sujeta a ciertos requisitos los cuales están en función de la temática a estudiar, del grado de conocimiento del estudiante y otros.

\section{Estructura de un sistema hipermedia adaptativo (SHA)}

Los SHA se encuentran constituidos por los siguientes elementos: 1) Dispositivos de interacción con el usuario para la recogida de información explícita e implícita, 2) Procesos de filtrado y análisis de variables, 3) Motor de decisión y 4) Gestor de contenidos Buitrago (citado por Benigni y otros, ob.cit.).

1. Dispositivos de interacción con el usuario para la recogida de información explícita e implícita. En relación a la obtención de información explícita se utilizan formularios, menús de opciones, botones, banners y barras de selección. En tanto que para la recogida de información implícita se hace uso de scripts que monitorizan el seguimiento de las actividades: detectores de clics, seguimiento de vínculos internos y externos.

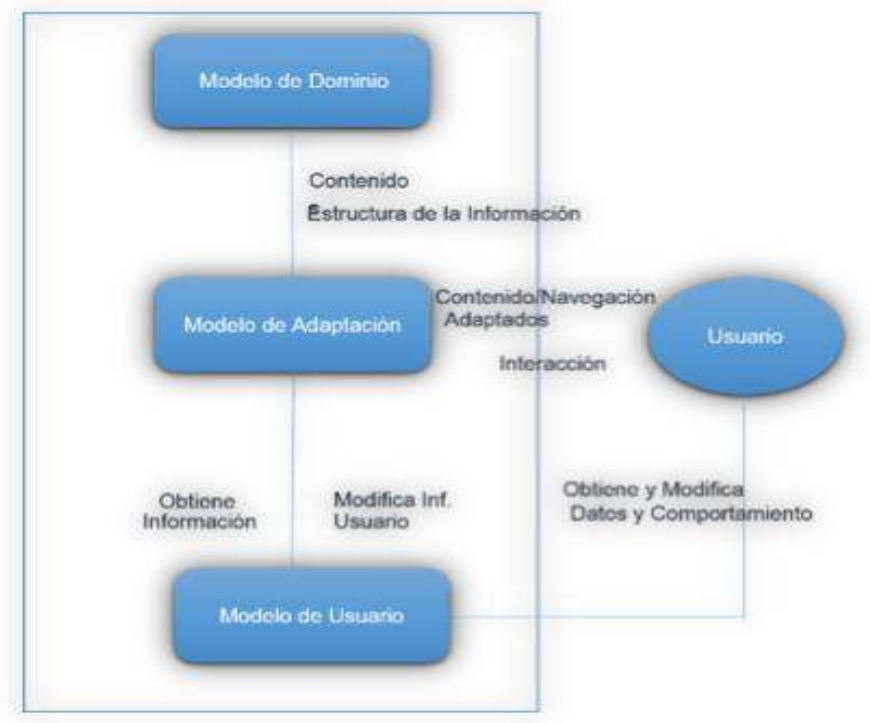

Gráfico1. Diagrama de los componentes de un SHA. Tomado de Sistemas hipermedia Adaptativos (SHA) por Buitrago (citado por Benigni y otros, ob.cit.).

2. Los Procesos de filtrado y análisis de variables. Consisten en complejos algoritmos que tienen como fin el análisis de las características y preferencias del usuario. El dispositivo 
SISTEMA HIPERMEDIA ADAPTATIVO PARA EL APRENDIZAJE DE BÚSQUEDAS AVANZADAS DE INFORMACIÓN

Eduardo Martínez y Any Montero ( PP. 81-107)

puede construir una tabla de perfiles para la posterior asignación de variables a procesos, objetos y contenidos. En el presente trabajo, estos procesos se basarán en el modelo de estilos de aprendizaje de Felder y Silverman.

3. Motor de decisión. Analiza las variables de entorno y los perfiles de usuario para decidir qué tipos de contenidos y en que formato se mostrarán ante una determinada petición de acceso. Para la implementación de este motor

4. Gestor de contenidos. Su función es construir y entregar los contenidos al usuario final. En el caso de páginas web dinámicas, estos serán elaborados a partir de multitud de elementos atómicos almacenados en bases de datos. Si se trata de un sitio estático el gestor procederá a elegir una entre las diferentes versiones de páginas almacenadas.Para el presente trabajo se diseñara de forma dinámica considerando los elementos multimedia almacenados den la base de datos del software educativo.

\section{Elementos adaptables de un sistema hipermedia adaptativos (SHA)}

Brusilovsky (citado por Benigni y otros, ob.cit.) define los siguientes elementos que se pueden adaptar en un SHA: 1) El conocimiento, 2) los objetivos y 3) las características del usuario.

1. El conocimiento. El SHA debe encontrarse al tanto en relación al progreso en el conocimiento del usuario para actualizar la información que mantiene sobre él mismo. En este sentido, se emplea un modelo representado como una red de conceptos y relaciones. En donde, cada concepto se almacena una estimación del nivel del conocimiento del usuario y, con base a esto, el sistema realiza la adaptación correspondiente.

2. Los objetivos. Se encuentran relacionado más con la información que busca el usuario en el hiperespacio, y no con el usuario como individuo. En otras palabras, un usuario de un hipermedia educativo puede tener como objetivo encontrar un material complementario del tema de estudio. Para el presente diseño estos se organizarán de acuerdo con el contenido de estudio sobre las búsquedas avanzadas de información en Internet.

3. Características del usuario. Información relevante y relacionada con la experiencia del usuario fuera del tema del SHA, como profesión, experiencia de trabajo en áreas relacionadas, punto de vista, perspectivas, entre otras. Asimismo, el diseño a implementar tomará en cuenta la información de su estilo de aprendizaje en el ámbito educativo. 
SISTEMA HIPERMEDIA ADAPTATIVO PARA EL APRENDIZAJE DE BÚSQUEDAS AVANZADAS DE INFORMACIÓN $\quad$ EN INTERNET Eduardo Martínez y Any Montero ( PP. 81-107)

\section{Ventajas en el ámbito educativo}

En las prácticas educativas los SHAE se conectan en todo momento con los intereses del estudiante, es decir, con lo que considera útil, de forma que las actividades de aprendizaje no terminan siendo aisladas. Por lo tanto, el proceso resulta motivador ya que el participante aprende los contenidos que le son relevantes, adaptándose a la capacidad de aprendizaje del mismo. Es decir, se le permite todo el tiempo precisar los contenidos a adquirir, así como también, responder a las preguntas planteadas, respetando su ritmo de trabajo y sin requerir la presencia constante del profesor, el cual sólo intervendrá cuando sea necesario. (Molina, y Damian, 2006).

En este orden de ideas, los aspectos visuales tomados en cuenta por el SHAE son muy atractivos para el estudiante, pues al permitir leer la información en un orden no secuencial le proporciona una mayor libertad para navegar. Es la libertad de navegación, lo que le conlleva a decidir el flujo de su aprendizaje. De este modo el estudiante puede dirigir su aprendizaje hacia los conceptos y aspectos de mayor interés para él, profundizando en las materias que necesite dominar, al mismo tiempo que descarta las ya estudiadas (Medina, García, Rodríguez y Parets, 2002).

La presentación adaptativa se refiere a la adaptación dentro de los contenidos de aprendizaje, a partir de las necesidades y habilidades de los usuarios. La idea de presentación adaptativa, es la de personalizarlos contenidos de los cursos, para moldearlos a las características específicas de los estudiantes, generando modelos de usuarios. En este sentido, consiste en tratar el contenido a ser presentado al usuario dependiendo de sus objetivos y de la base que éste posea del conocimiento manejado por el sistema. A partir de estas informaciones el sistema presentará el conocimiento que es relevante al usuario o que el mismo está listo para recibir (Martí, 2009).

Asimismo, los SHA solucionan los problemas orientación y pérdida del alumno, propios de los sistemas hipermedia tradicionales, adaptando el hiperespacio disponible dependiendo del conocimiento del estudiante. A medida que éste va aprendiendo, la accesibilidad crece, dándole la oportunidad de alcanzar nuevas informaciones. Además, se pueden incluir estructuras y patrones conocidos para representar la información con la que se trabaja y se pueden añadir herramientas de ayuda a la navegación. También, evitan que la información sea estática intentando adaptar la información, o la presentación de esa información a las características, 
SISTEMA HIPERMEDIA ADAPTATIVO PARA EL APRENDIZAJE DE BÚSQUEDAS AVANZADAS DE INFORMACIÓN Eduardo Martínez y Any Montero ( PP. 81-107)

preferencias y necesidades del usuario Molina, y Damian,ob.cit.).

Este proceso de personalización permite mostrar el contenido que es apropiado para el grado de conocimiento de cada estudiante, ajustando las ayudas, los mensajes de error y las tareas que ofrece al mismo (Grimón, 2008).Es importante señalar que para la presente investigación se adoptará la teoría adaptativa propuesta por Brusilovsky citado por Barragán, 2008), tomando en cuenta exclusivamente los estilos de aprendizaje.

\section{METODOLOGÍA}

La presente investigación se ubicó dentro del paradigma positivista, conforme a lo expresado por Hurtado y Toro (2007), se entiende como "la investigación que, predominantemente, tiende a usar instrumentos de medición y comparación que proporcionan datos cuyo estudio requiere el uso de modelos matemáticos y de la estadística” (p.39).

Asimismo, el trabajo consistió en el diseño de un Sistema Hipermedia Adaptativo para el aprendizaje de búsquedas avanzadas de información en Internet. Se fundamentó en la modalidad de Proyecto Especial que de acuerdo con el Manual de Trabajos de Grado de Especialización y Maestría y Tesis Doctorales (UPEL, 2012) define como:

Creaciones tangibles, susceptibles de ser utilizadas como soluciones a problemas demostrados, o que respondan a necesidades e interés de tipo cultural. Se incluyen en esta categoría los trabajos de elaboración de libros de texto y de materiales de apoyo educativo, el desarrollo de software, prototipos y de productos tecnológicos en general (p. 22).

De acuerdo con los objetivos del estudio, en su fase diagnóstica se apoyó en una modalidad de investigación de campo transeccional con un nivel de complejidad descriptivo, que de acuerdo con Arias (2006) "consiste en la especificación de un hecho o fenómeno, individuo o grupo, con el fin de establecer su estructura o comportamiento" (p.25). En otras palabras, se observa y cuantifica el cambio de una o más características, sin establecer relaciones entre éstas.

\section{Diseño de la Investigación}

La labor de indagación se ejecutó en tres fases claramente definidas con el fin de proporcionar respuestas a los objetivos planteados de la investigación. Durante la primera fase se hizo referencia a un análisis situacional que facilitó el diagnóstico de la necesidad de la elaboración del Sistema Hipermedia Adaptativo para el aprendizaje de búsquedas avanzadas de 
SISTEMA HIPERMEDIA ADAPTATIVO PARA EL APRENDIZAJE DE BÚSQUEDAS AVANZADAS DE INFORMACIÓN

Eduardo Martínez y Any Montero ( PP. 81-107)

información en Internet. La segunda fase incluyo lo referente a su diseño, y por último, la tercera fase consistió en el proceso de validación tecnológica, y pedagógica efectuado por los expertos, asimismo, la llevada a cabo por parte de los usuarios.

\section{Población y muestra}

Arias (ob.cit.), afirma que la población es un conjunto finito o infinito de elementos con características comunes para los cuales serán extensivas las conclusiones de la investigación. Además, define la población finita como "una agrupación en la que se conoce la cantidad de unidades que la integran, además de existir un registro documental de dichas unidades” (p.82). Con referencia a lo anterior, una población es el conjunto de elementos que concuerdan con una serie determinada de especificaciones, por lo que en esta fase de diagnóstico se consideró como población de estudio todos los estudiantes inscritos en el primer 1er semestre de Ingeniería Agronómica de la UCLA.

Mientras que la muestra se define como "un subconjunto representativo y finito que se extrae la población accesible" (Arias, ob.cit, p.83). Por otra parte, Tamayo (2004) considera que esta se toma a partir de la población, cuando no es posible estudiar cada uno de los individuos de la misma. Además, el mencionado autor considera que es representativa de la población porque "descansa en el principio de que las partes representan al todo y, por tal, refleja las características que definen la población de la que fue extraída, lo cual nos indica que es representativa" (p.176).

En cuanto al cálculo del tamaño de la muestra, estese basó en la propuesta de Hurtado y Toro (ob.cit.) en relación a las investigaciones descriptivas, en donde establece que "requieren de muestras que abarquen al menos el diez por ciento (10\%) de la población para que sus resultados puedan considerarse como válidos" (p.92).

El proceso de selección de la muestra se implementó al azar mediante un sorteo, en donde a cada estudiante del 1er Semestre de Ingeniería Agronómica se le asignó un número que variará desde uno (1) hasta la cantidad total de la muestra, que se generó aleatoriamente mediante la serie de funciones incluidas para trabajar con distribuciones de probabilidad, en particular para generar números aleatorios del paquete "random" de la herramienta estadística de Software Libre "R Language". 
SISTEMA HIPERMEDIA ADAPTATIVO PARA EL APRENDIZAJE DE BÚSQUEDAS AVANZADAS DE INFORMACIÓN $\quad$ EN INTERNET Eduardo Martínez y Any Montero ( PP. 81-107)

\section{Técnica e instrumento de recolección de datos}

Para los intereses del presente proyecto se aplicó la encuesta como técnica de recolección de datos, esta se define como "una técnica que pretende obtener información que suministra un grupo o muestra de sujetos acerca de sí mismos, o en relación a un tema en particular" (Arias, 2006., p.72). La información obtenida se registró en un cuestionario, siguiendo con el autor, es un estilo de encuesta que se efectúa de manera escrita mediante un formato en papel contentivo de una serie de preguntas. Además, se le conoce como cuestionario auto administrado debido que debe ser llenado por el encuestado, sin participación del encuestador. .

\section{Fase 2: Diseño}

Esta fase se relaciona con el segundo objetivo, el cual consiste en diseñar un Sistema Hipermedia Adaptativo para el aprendizaje de búsquedas de información en Internet. Durante la elaboración de este Software Educativo se consideró no solo conceptualmente el aprendizaje, sino cómo se desarrolla tal proceso. Para la presente investigación, se utilizó la metodología de trabajo MOOMH, Metodología Orientada a Objetos para desarrollar software Multimedia e Hipermedia presentada por Benigni (2004).

La MOOMH contribuye al diseño de aplicaciones hipermedias educativas por medio de una combinación de herramientas de Ingeniería del Software (metodologías, técnicas) que se exponen de manera precisa a través de cuatro (4) modelos:1) Modelo de Requerimiento, 2) Modelo de Análisis, 3) Modelo de Diseño y 4) Modelo de Implementación. La característica principal de estos, es que se encuentran interrelacionados, permitiendo que al mismo tiempo que la comunicación sea iterativa entre cada una de las mismas, por lo que la interacción existente entre ellos sirve de base para el desarrollo de aplicaciones educativas e informativas para la Web (Benigni, ob.cit).

En relación con la primera fase, el Modelo de Requerimientos, como fundamento central de la metodología se determinó hacia quien iba dirigido el software a desarrollar, lo que comprendió la determinación de requerimientos del problema (Benigni, ob.cit.). Asimismo, se incluyó el Modelo de Domino como uno de los componentes en el diseño de los Sistemas Hipermedias Adaptativos (SHA).

Siguiendo con la segunda fase, el Modelo de análisis, se procedió a modelar las tareas de los diferentes usuarios a través de la identificación de los objetos, que se definieron por medio 
SISTEMA HIPERMEDIA ADAPTATIVO PARA EL APRENDIZAJE DE BÚSQUEDAS AVANZADAS DE INFORMACIÓN Eduardo Martínez y Any Montero ( PP. 81-107)

de todos los tópicos de contenido relacionados con los temas a desarrollar para cumplir con los objetivos de aprendizaje. Para esto se contó con la elaboración del mapa de navegación del sistema, el cual se representó a través de una guía de navegación donde se especificó el nivel de profundidad de los enlaces para cada una de las lecciones. Por último, el diseño de los objetos, en el cual, se procedió en función de los elementos multimedia: video, texto, animación, imagen, sonido, entre otros a representar de una manera sencilla los temas de estudio.

Durante el tercer momento, el Modelo de diseño, se prosiguió con la elaboración del prototipo de la interfaz, asimismo, se le adiciono el primeramente el Modelo de Usuario y el Modelo de Adaptación. En relación al primero, se almaceno aspectos relevantes del usuario como preferencias de aprendizaje, además de la interacción que realiza éste con el sistema; permitiendo así que el segundo, en base a lo que se encuentra almacenado, adapte la información o contenido a mostrar de acuerdo a cada usuario.

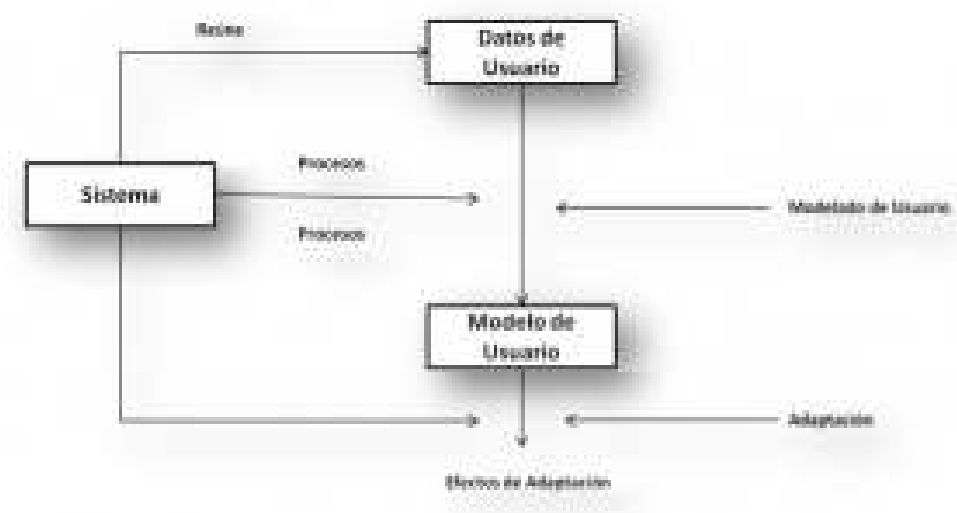

Gráfico 2.Ciclo clásico modelado de usuario en sistemas adaptativos. Tomado de "MOOMH: Metodología Orientada a Objetos Multimedia e Hipermedia" Benigni y Marcano (ob.cit.).

Por último, durante la fase del Modelo de implementación, se desarrolló la aplicación utilizando lenguaje de programación C\# basado en el entorno de desarrollo Microsoft Visual Studio .NET 2013 Update 2. Además, en su etapa final, se aplicó una prueba piloto que sometió a depuración del software educativoa partir de una muestra representativa de los estudiantes del 1er semestre de Ingeniería Agronómica de la UCLA. Asimismo, se determinó en una práctica 
SISTEMA HIPERMEDIA ADAPTATIVO PARA EL APRENDIZAJE DE BÚSQUEDAS AVANZADAS DE INFORMACIÓN $\quad$ EN INTERNET Eduardo Martínez y Any Montero ( PP. 81-107)

real la efectividad de la aplicación en relación a la satisfacción de las necesidades y funcionalidades requeridas.

\section{Fase 3: Validación}

La incorporación del Software Educativo en el desarrollo de actividades pedagógicas requiere de un soporte científico de validación, que sirva de garantía para el logro de sus propósitos académicos. Para Fallas y Chavarría (2010), este proceso consiste en la determinación del grado de adecuación de los respectivos programas al contexto educativo. Por lo tanto, Cova, Arrieta y Duran (2008) proponen que se deben prever dos tipos de validación, una para la fase de desarrollo donde se aplica el método de criterio de expertos, y otra para la fase de puesta en práctica en la que se emplea validación de campo a través de un contacto directo con los posibles usuarios del sistema. Para este estudio, se consideró tanto para la primera como segunda validación las orientaciones dispuestas Marqués (2009) en sus instrumentos,el cual a su vez propone la aplicación de los mismos para recoger las apreciaciones de los especialistas en las dimensiones de contenido, diseño pedagógico, y técnicas.

En cuanto a la dimensión de contenido, se contó con tres (3) expertos pedagogos, los cuales, a través del instrumento de validación de contenido, validaron que el software educativo fuese relevante con respecto a: redacción, lenguaje adecuado al nivel de enseñanza, vigencia científica, confiabilidad conceptual, pertinencia, demostraciones, transferencia de aprendizaje, motivación, confiabilidad psicopedagógica, y refuerzos.

En cuanto a la validez del diseño pedagógico, se continuó adaptando el modelo propuesto por Marqués (ob.cit.), el instrumento constó de doce (12) aspectos: presentación del software, videos, color, texto, imágenes, sonido, objetivos, secuencia lógica, flexibilidad, diseño pedagógico, interacción y evaluación, con un total de quince (15) ítems.

De la misma manera, para la dimensión técnica se contó con tres (3) expertos en Informática, el cual por medio del Instrumento de validación técnica, sometió a juicio el software educativo a través de los siguientes aspectos: estructura de programación, facilidad de comprensión, adaptabilidad, interfaz gráfica y confiabilidad. Esta a su vez, consistió en verificar que todas y cada una de las funciones previstas en el diseño del Software se hayan desarrollado en forma pertinente. 
SISTEMA HIPERMEDIA ADAPTATIVO PARA EL APRENDIZAJE DE BÚSQUEDAS AVANZADAS DE INFORMACIÓN

Eduardo Martínez y Any Montero ( PP. 81-107)

En cuanto a la validación de campo, se desarrolló durante la prueba piloto con los posibles usuarios del sistema, a través de un instrumento este a su vez permitió establecer si efectivamente el software educativo en cuestión cumplía con el propósito previsto, es decir, satisface la necesidad educativa, permitiendo brindar la solución del problema de aprendizaje que origino el desarrollo del estudio.

\section{ANÁLISIS E INTERPRETACIÓN DE LA INFORMACIÓN}

El proceso de análisis e interpretación de la información consiste en el recuento, clasificación y ordenación de los datos en cuadros y gráficos, a fin de explicarlos de manera que queden claros los resultados obtenidos en la investigación (Hurtado y Toro, 2007).El proceso se desarrolló con base a la información recopilada por medio del cuestionario de escala de múltiples alternativas de respuesta, el cual se aplicódurante el proceso de investigación, en función del estudio y los objetivos establecidos.

Es de hacer notar que en cuanto a la necesidad del diseño del software educativo se presentaron 5 dimensiones: (a) Las TIC en el campo educativo; (b) El software educativo; (c) La hipermedia adaptativa; (d) Los estilos de aprendizaje y las búsquedas avanzadas de información, es importante mencionar que en las cinco dimensiones se encontró que entre el 85 y $90 \%$ de la población considero necesario y muy necesario el desarrollo de un software educativo adaptativo que responda a los estilos de aprendizaje y coadyuve en la búsqueda de información asertiva, como puede ser apreciado en el siguiente gráfico:

\section{Necesidad del Diseño del Software Educativo}

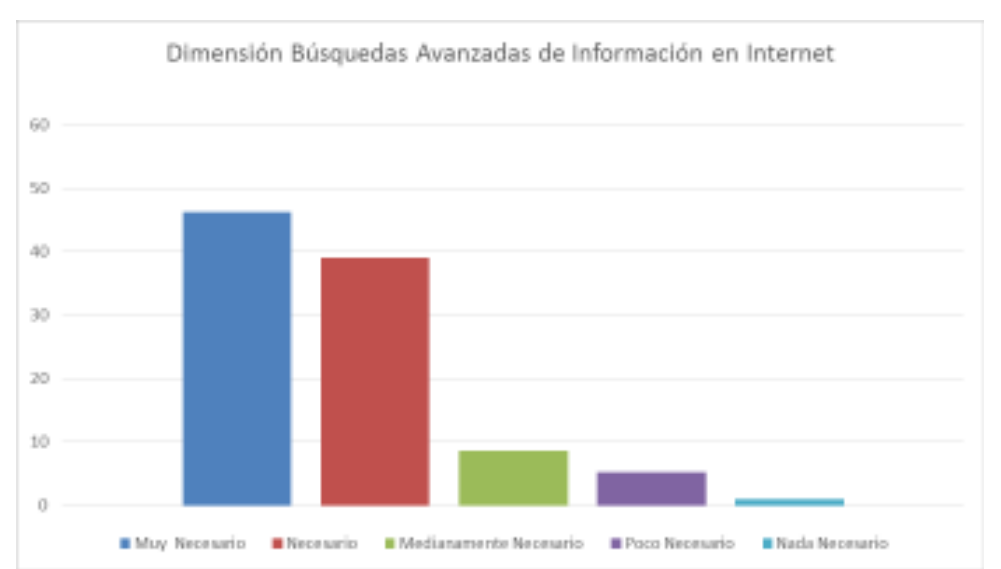

Gráfico 3.Percepción global de la muestra de la necesidad del Diseño software educativo adaptativo 
SISTEMA HIPERMEDIA ADAPTATIVO PARA EL APRENDIZAJE DE BÚSQUEDAS AVANZADAS DE INFORMACIÓN $\quad$ EN Eduardo Martínez y Any Montero ( PP. 81-107)

\section{Resultados de la fase 2: Diseño del Software Educativo}

Durante el diseño y elaboración de un Software Educativo, además de los aspectos técnicos, es importante considerar el cómo se aprende, por tal motivo, durante el presente trabajo se aplicó la Metodología Orientada a Objetos Multimedia e Hipermedia (MOOMH) de Benigni (2004). Esta, a su vez permitió diseñar la aplicación hipermedia educativa acorde con los estándares pedagógicos y tecnológicos del software educativo. A continuación se exhiben algunas pantallas del diseño:
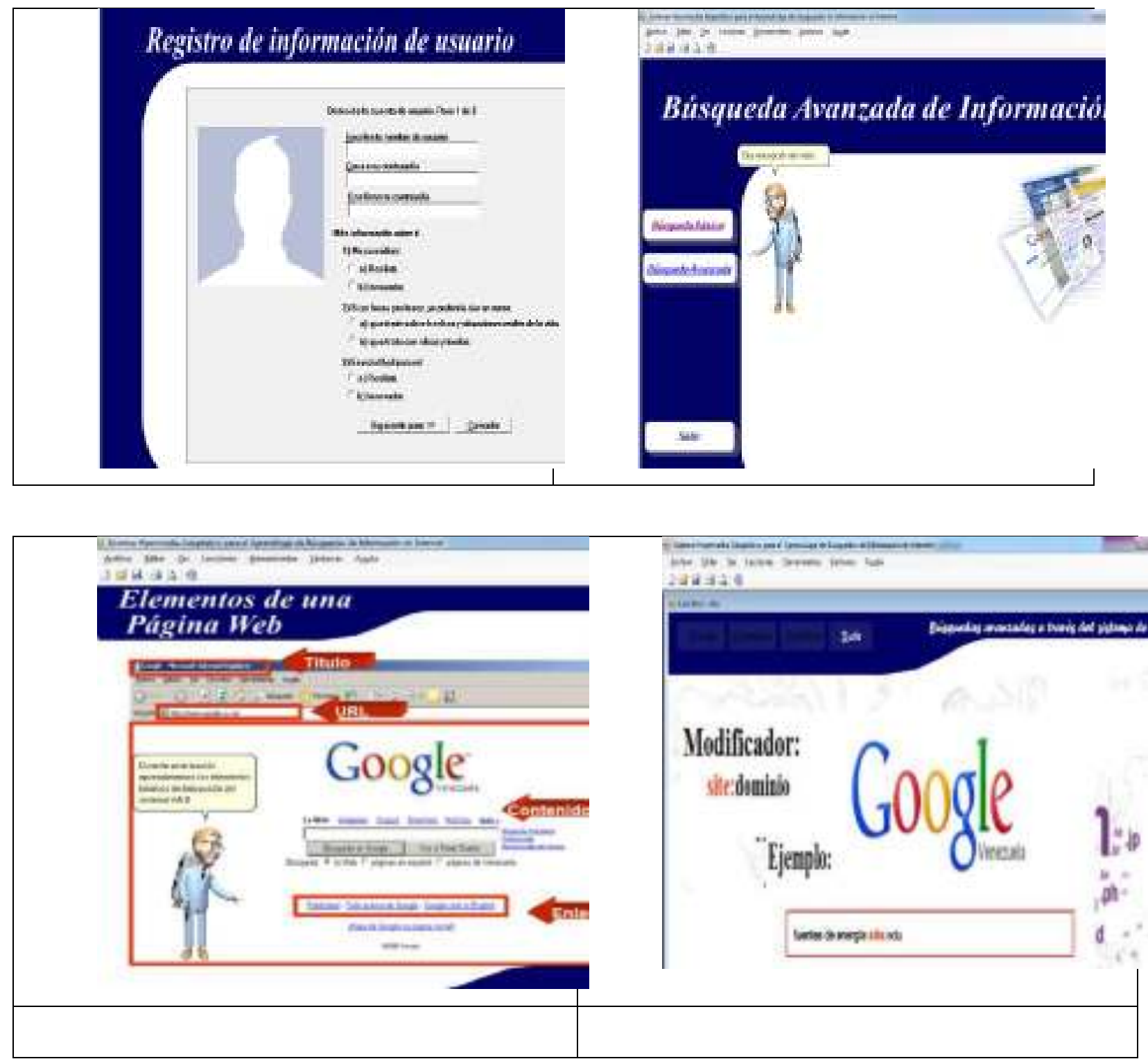
SISTEMA HIPERMEDIA ADAPTATIVO PARA EL APRENDIZAJE DE BÚSQUEDAS AVANZADAS DE INFORMACIÓN $\quad$ EN INTERNET

Eduardo Martínez y Any Montero ( PP. 81-107)

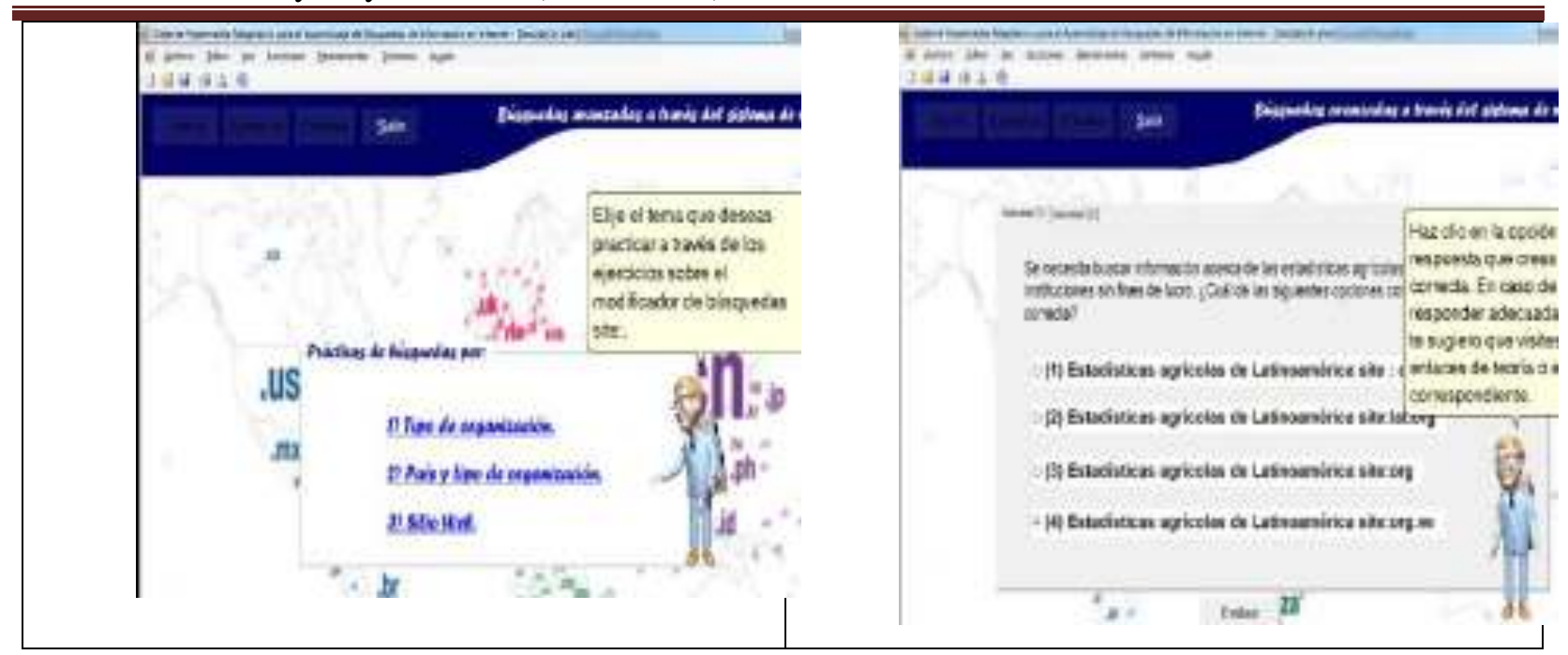

Por otra parte, en la fase de validación se pudo corroborar que el $90 \%$ de los validadores consideró que el software educativo adaptativo cumple con los aspectos técnicos, de contenido y de diseño. De esta manera se ve garantizado que el prototipo cumple con las características esenciales para que este sirva de herramienta idónea en el apoyo de los estudiantes objeto de estudio y de la población en general. Esta información puede ser apreciada en el gráfico en la apreciación de los potenciales usuarios:

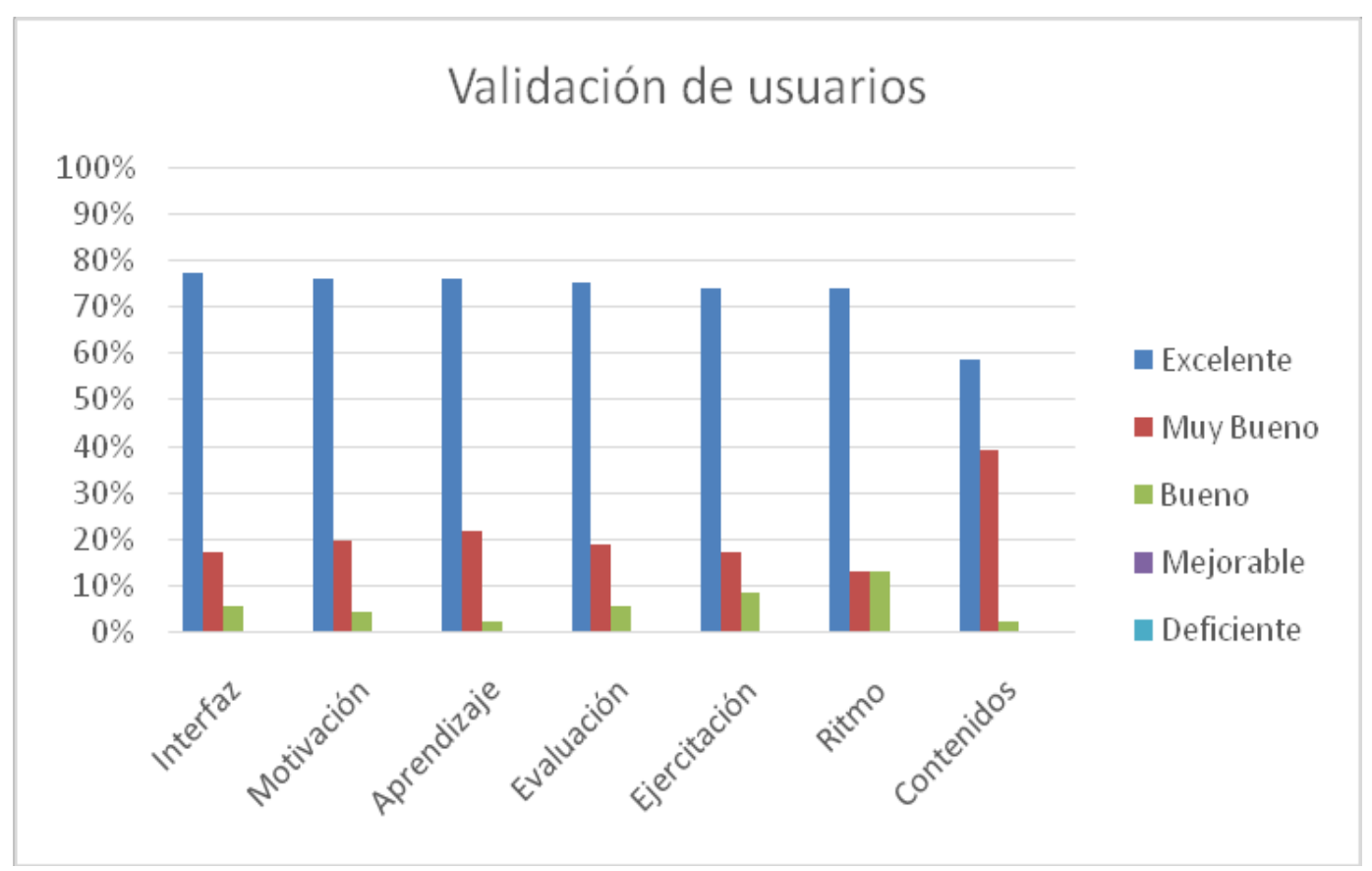

Gráfico 5. Percepción global de los usuarios. 
SISTEMA HIPERMEDIA ADAPTATIVO PARA EL APRENDIZAJE DE BÚSQUEDAS AVANZADAS DE INFORMACIÓN $\quad$ EN INTERNET Eduardo Martínez y Any Montero ( PP. 81-107)

\section{CONCLUSIONES}

El presente estudio tuvo como propósito el diseño de un Software Educativo para el aprendizaje de búsquedas avanzadas de información en Internet, atendiendo a la problemática presentada por los estudiantes objeto de estudio, en cuanto a la forma de localizar la información en Web, ya que existen debilidades y limitaciones en la obtención de recursos académicos en su contexto educativo, ya que, estos resultan escasos, de fuentes no confiables, descontextualizados, o de poca vigencia, por lo tanto, no pertinentes para su uso académico.

En el primer objetivo, se diagnosticó la necesidad de diseño del respectivo Software Educativo, a través de los cuestionarios aplicados a treinta (30) estudiantes pertenecientes a la muestra de estudio. Este proceso empírico basado en las dimensiones teóricas de las TIC, Software Educativo, Hipermedia Adaptativo, Estilos de aprendizaje, Búsquedas Avanzadas de información; arrojo como resultado, que el 87,2\% de los consultados consideraron entre muy necesario y necesario el uso del Sistema Hipermedia Adaptativo para el Aprendizaje de Búsquedas Avanzadas de Información en Internet. Por lo que, este alto porcentaje de apreciaciones corrobora lo aportado por los referentes del marco teórico del presente estudio a favor del uso del Software Educativo, otorgándole de este modo la pertinencia científica al presente estudio.

Por otra parte, el segundo objetivo, se continuó con el diseño del Software Educativo, considerando los requerimientos de los estudiantes consultados, y los aportes teóricos estudiados. En este sentido, entre las alternativas metodológicas que sustentan el diseño instruccional, se eligió la denomina MOOMH, Metodología Orientada a Objetos para desarrollar software Multimedia e Hipermedia, formulada por Benigni (ob.cit.), ya que sus etapas favorecen un proceso de desarrollo iterativo e incremental, lo que garantiza la producción de un software en correspondencia con los objetivos especificados de la investigación.

En este orden de ideas, el diseño del software educativo como medio instruccional para el aprendizaje de las Búsquedas avanzadas de Información en Internet, permitió brindar una alternativa de solución al problema planteado, presentando el contenido teórico-práctico en formato digital. Por tanto, se obtuvo un medio instruccional acompañado de una presentación, que se caracterizó por ser amigable, personalizada, contentiva de: imágenes, videos, colores, fondos musicales, autoevaluaciones y reforzamiento, entre otras; destacando que cada una de 
SISTEMA HIPERMEDIA ADAPTATIVO PARA EL APRENDIZAJE DE BÚSQUEDAS AVANZADAS DE INFORMACIÓN $\quad$ EN INTERNET

Eduardo Martínez y Any Montero ( PP. 81-107)

estos elementos se expuso de forma cónsona con el proceso de aprendizaje de los tiempos actuales.

Por último, en el tercer objetivo, se efectuó la fase de validación de expertos y de usuarios, se trabajó con la planteada por Marqués (2009), entre la gran variedad de modelos y pautas de evaluación de software educativos; debido a que recoge los rasgos fundamentales del software educativo, por lo cual, a través de las características más resaltantes que presenta este modelo de evaluación, se sometió a juicio de especialistas y posibles usuarios del sistema, los criterios de calidad pertinentes con la problemática planteada de la presente investigación.

En este sentido, los expertos en las áreas de contenido, diseño pedagógico e Informática, determinaron la validez del software educativo, al cumplir los requisitos mínimos de un material multimedia educativo computarizado. Asimismo, la validación de usuarios llevada a cabo a través de los usuarios encuestados durante la prueba piloto, corroboró que él mismo puede ser utilizado por los estudiantes del 1er semestre de Ingeniería Agronómica de la UCLA como herramienta de aprendizaje del tema de interés.

\section{REFERENCIAS}

Arias, F. (2006). El Proyecto de Investigación. Introducción a la metodología científica (5a. ed.). Caracas: Episteme, C.A.

Baena, J. (2008). Las TICs Un nuevo recurso en el aula [Documento en línea]. Disponible: http://www.csi-

csif.es/andalucia/modules/mod_ense/revista/pdf/Numero_13/JUAN_J_BAENA_1.pdf [Consulta:2014, Noviembre 21].

Benigni, G. (2004). Una Metodología Orientada a Objetos para la Producción de Software Multimedia [Revista en línea], SABER, (16) 1 pag. 26-32. Disponible: http://www.urbe.edu/UDWLibrary/ArticulosAdvance.do?operator=EMPTY\&tag=100\&wo rd=Benigni,\%20Gladys.

Benigni, G., y Celada, J. (2008). Moomh Case. Herramienta Automatizada para la ayuda al Desarrollo de Sistemas Hipermediales [Revista en Línea] Facultad De Ingeniería U.C.V., (23) 4 ,

99-110.

Disponible: http://saber.ucv.ve/ojs/index.php/rev_fiucv/article/view/5082/4889 [Consulta: 2015, Enero 20].

Benigni, G., y Marcano, I. (2014). ¿Qué herramientas utilizar para diseñar sistemas hipermedia educativo adaptativos? [Revista en línea] Espacios, 35 (6), pág. 13 . Disponible:http://www.revistaespacios.com/a14v35n06/14350613.html [Consulta: 2015, Enero 27]. 
SISTEMA HIPERMEDIA ADAPTATIVO PARA EL APRENDIZAJE DE BÚSQUEDAS AVANZADAS DE INFORMACIÓN

Eduardo Martínez y Any Montero ( PP. 81-107)

Benigni, G., y Marcano, I. (2014). Análisis de Alternativas Metodológicas para el Desarrollo de Software Educativo [Revista en línea] Saber, Universidad de Oriente, Venezuela. (26) 3. pag. 297-304. Disponible:http://www.revistaespacios.com/a14v35n06/14350613.html [Consulta: 2015, Enero 12].

Berlanga, A. (2006). Diseños Instructivos Adaptativos: Formación Personalizada y Reutilizable en Entornos Educativos. [Documento en línea]. Tesis Doctoral no publicada, Universidad de Salamanca, Departamento de Informática y Automática Facultad de Ciencias.

Disponible: http://gredos.usal.es/jspui/bitstream/10366/21858/1/TD_Disenos\%20instructivos.pdf [Consulta: 2013, Junio 15].

Calderín, M., y Rojano, M. (2006). Estrategias y herramientas de búsqueda de información en la web para periodistas [Documento en línea]. Disponible:http://www.ucab.edu.ve/tl_files/CIC/recursos/Period_dig_Doc.pdf [Consulta: 2013, Mayo 02].

Cañellas, A. (2006). Impacto de las TIC en la educación: un acercamiento desde el punto de vista de las funciones de la educación. Quaderns digitals, Revista de Nuevas Tecnologías y Sociedad [Revista en línea], $43 . \quad$ Disponible: http://dialnet.unirioja.es/servlet/articulo?codigo=2037601\&orden=72732\&info=link [Consulta: 2013, Junio 8].

Cova, Á., Arrieta, X., y Duran, J. (2008). Revisión de Modelos para Evaluación de Software Educativos [Revista en Línea] Télématique URBE, (7) 1. Disponible: http://publicaciones.urbe.edu/index.php/telematique/article/viewFile/900/2233 [Consulta: 2015, Febrero 10].

Grimón, F. (2008). Modelo para la Gestión de Dominios de Contenido en Sistemas Hipermedia Adaptativos Aplicados a Entornos de Educación Superior Semipresencial. [Documento en línea]. Tesis Doctoral no publicada Universidad Politécnica de Cataluña, Programa de Doctorado en Ingeniería $\quad$ Multimedia. Disponible http://www.tesisenred.net/bitstream/handle/10803/6551/01Fgm01de01.pdf?sequence=1 [Consulta: 2013, Mayo 05].

Grimón, F., Guevara, M., y Monguet, J. (2010). Influencia de usar un Sistema de Hipermedia Adaptativo (SHA) en la modalidad de Aprendizaje Combinado (Blended Learning). ANALES [Revista en línea], (10)1, 93-112. Disponible: http://andromeda.unimet.edu.ve/ojs/index.php/ra/article/view/20. [Consulta: 2013, Marzo $10]$.

Hernández, R., Fernández, C., Baptista, P. (2014). Metodología de la investigación. (6a, ed.). México: McGraw-Hill.

Marquès, P. (2007). Impacto de las TIC en la Enseñanza Universitaria [Documento en línea]. Departamento de Pedagogía Aplicada, Facultad de Educación, UAB. Disponible:http://ddd.uab.cat/pub/dim/16993748n11a5.pdf. 
SISTEMA HIPERMEDIA ADAPTATIVO PARA EL APRENDIZAJE DE BÚSQUEDAS AVANZADAS DE INFORMACIÓN $\quad$ EN

Eduardo Martínez y Any Montero ( PP. 81-107)

Marquès, P. (2009). Entornos Formativos Multimedia: Elementos, Plantillas de Evaluación/Criterios de Calidad [Documento en línea]. Disponible: http://www.peremarques.net/calidad.htm [Consulta: 2015, Febrero17].

Martí, J. (2009). Una mirada al desarrollo, evolución y tendencias de los sistemas inteligentes adaptativos en el ámbito educativo [Documento en línea] . Disponible: http://www.readbag.com/monografias-trabajos-pdf4-estilos-aprendizaje-y-modelo-usuarioestilos-aprendizaje-y-modelo-usuario [Consulta: 2014, Diciembre 10].

Ribaya, F. J. (2007). Las Tutorías y las TICS Universidad Alfonso X EL SABIO, Alcalá Henares. [Documento en línea]. Disponible:http://www3.udenar.edu.co/viceacademica/proy_men/ABRIL/UVIRTU.doc [Consulta: 2013, Mayo 10].

Universidad Pedagógica Experimental Libertador, Vicerrectorado de Investigación y Postgrado 4ta Edición. (2012). Manual de trabajos de grado de especialización y maestría y tesis doctorales. Caracas: Autor. 